\title{
Chemical Peeling as an Innovative Treatment Alternative to Oral Antifungals for Onychomycosis in Special Circumstances
}

\author{
Sidharth Sonthalia ${ }^{a}$ Deepak Jakhar ${ }^{b}$ Pravesh Yadav ${ }^{c}$ Ishmeet Kaur ${ }^{d}$ \\ a SKINNOCENCE: The Skin Clinic \& Research Centre, Gurugram, India; b NDMC Medical College \& Hindu Rao Hospital, \\ Department of Dermatology, New Delhi, India; ' Department of Dermatology \& STD, Lady Hardinge Medical College \\ \& Associated Hospitals, New Delhi, India; ${ }^{d}$ ESI-PGIMSR, Department of Dermatology \& STD, New Delhi, India
}

\section{Established Facts}

- Toe nail onychomycosis is a common condition in elderly and diabetic patients.

- Oral antifungals are best avoided in such patients who often have comorbidities.

- Topical antifungal agents are largely ineffective as monotherapy.

\section{Novel Insights}

- Regular chemical peeling of nails combined with topical antifungal nail lacquers can be a potential curative approach.

- Peeling agents like acetic acid, salicylic acid, jasmonic acid, biosulphur, and potassium iodide - components of Black Peel - have broad-spectrum antimicrobial, specifically antifungal and anti-biofilm properties.

- Nail peeling with Black Peel can be combined with topical antifungal medications for augmented results.

\section{Keywords}

Dermatophyte $\cdot$ Nail peeling $\cdot$ Black peel $\cdot$ Acetic acid .

Onychomycosis

\begin{abstract}
Toe nail onychomycosis (ONM) is a common problem in elderly patients. Treatment in diabetic patients with associated comorbidities such as congestive heart failure becomes a challenge. Oral antifungals are best avoided owing to most of them being contraindicated or unsafe due to comorbidi-
\end{abstract}

ties and/or risk of drug interactions stemming from polypharmacy. Topical agents alone are not effective. Unlike isolated ONM in healthy and younger patients, ONM involving multiple nails in geriatric and diabetic patients warrants treatment as it serves as a source of recurrent tinea of the feet and other sites, and even contributes to formation of diabetic foot ulcers. Long-pulsed Nd:YAG and fractional $\mathrm{CO}_{2}$ laser (in addition to antifungals) have been reported to provide gratifying results in toe nail ONM. But high treatment cost is a major limitation. Nail peeling with glycolic acid (GA) and low-concentration croton-oil free phenol (CFP) peels
(C) 2019 S. Karger AG, Basel

E-Mail karger@karger.com

www.karger.com/sad
Deepak Jakhar

NDMC Medical College, Department of Dermatology

Hindu Rao Hospital

New Delhi 110007 (India)

E-Mail dr.deepakjakhar@yahoo.in 
have been used in different nail conditions but not specifically for ONM. We report the successful use of Black peel (a superficial-to-medium depth peel used typically for anti-acne and cosmetic indications), a combination of $50 \%$ black acetic acid, $0.5 \%$ salicylic acid, $6 \%$ tetrahydrojasmonic acid, $10 \%$ bio sulphur, and $0.1 \%$ potassium iodide, in two difficult cases with ONM of multiple nails, the rationale of use being the additional anti-fungal effects of all the peel components.

\section{Introduction}

Toe nail onychomycosis (ONM) in the elderly and patients with uncontrolled diabetes with neuropathy and other comorbidities like congestive heart failure (CHF), hepatic or renal insufficiency poses a therapeutic dilemma. Apart from being contraindicated or doubtful safety of oral antifungals in such situations, drug interactions that may arise due to patients' polypharmacy and strong impact of drugs like itraconazole on CYP isoforms constitute major impediments for considering oral antifungal agents in such patients [1]. Topical antifungal medications alone are a poor choice for ONM involving multiple toe nails owing to modest improvement and need for prolonged therapy. Physical therapies, especially photodynamic therapy (PDT) and laser treatment with longpulsed Nd:YAG and/or fractional $\mathrm{CO}_{2}$ lasers have recently been reported to provide good results in toe nail ONM when used in combination with topical and/or oral antifungals [2-4]. But high treatment cost, lack of adequate training of the healthcare provider, and patients' reluctance for a "laser" treatment constitute important limitations of this approach. Chemical peeling in nails has been used in the past $[5,6]$. However, the role and use of nail peeling specifically for ONM has not been reported. We explored the role of sequential nail peeling for toe nail ONM with Black peel, a combination of $50 \%$ black acetic acid (BAA), $0.5 \%$ salicylic acid (SA), and $0.1 \%$ potassium iodide (KI), in addition to $6 \%$ tetrahydrojasmonic acid (JA), and $10 \%$ bio sulphur (BS), otherwise popular as a superficial-to-medium depth peel for anti-acne and cosmetic indications. Our rationale of preferring Black peel over other peel combinations is attributable to the additional anti-fungal (as well as broad-spectrum anti-microbial) properties of all its components [7-11]. We report the successful use of sequential nail peeling with Black Peel in combination with daily topical ciclopirox (8\%) lacquer in two elderly diabetic patients in whom oral antifungals was not an option.

\section{Case Report}

Two elderly diabetic women with CHF (grade 2 and 3, respectively) and threatened diabetic foot ulceration and distolateral subungual ONM (DLSO) involving multiple toe nails (case 1 - three toe nails of left foot, with suspicion of impending near total dystrophy of second and third toe nail; case 2 - four toe nails of left foot with more clinical severity in first and second toe nails) revealed mixed infection on fungal culture - Trichophyton rubrum + Candida albicans in case 1, and C. albicans + Fusarium spp. in case 2, respectively. The second patient had been applying a topical steroidclotrimazole combination cream on the nail folds for the past 4 weeks (suggested by a local pharmacist), which had reduced the previously present inflammatory signs involving the cuticles and nail folds. While CHF ruled out oral itraconazole as a treatment option, potential adverse effects of prolonged high-dose oral terbinafine (due to obesity) and the limitation of terbinafine's weak activity against yeasts and molds eliminated that as an option as well. Both patients also refused to take oral treatment as well as laser treatment for ONM. Topical ciclopirox olamine (CPO) 8\% (nail lacquer) once daily at night was given to the patients but no improvement was noticed after 10 weeks of follow-up. Thus, we devised a protocol of continuing daily CPO 8\% nail lacquer application, while adding on periodic planned sessions of nail peeling using Black Peel for at least the next 6 months. After safeguarding the nail folds and cuticles with vaseline, a uniform coat of Black peel was applied over the infected toe nails (Fig. 1), followed by a second coat 20 min later. After 40 min, the peel was washed off with plain water followed by immediate application of $8 \%$ CPO lacquer. Peel sessions were performed weekly for 4 weeks and fortnightly for the next 4-6 months. Mycological evaluation with $40 \% \mathrm{KOH}$ examination of nail clipping and subungual debris was done fortnightly till it became negative (after 6 weeks and 10 weeks of starting the protocol, in case 1 and 2, respectively). Fungal culture was repeated every 4 weeks till it became negative (after 22 and 24 weeks of starting the protocol, in case 1 and 2 , respectively). The patients were advised daily self-application of $\mathrm{CPO}$ lacquer at night during the entire treatment period.

\section{Results}

Both patients started to show clinical improvement by the end of 4 weeks. Complete mycological cure was attained at the 6th month. Both patients were satisfied with the final visible appearance of the toe nails (Fig. 2). The patients reported no adverse effects during the treatment.

\section{Discussion}

Chemical peeling has been used on skin for a long time; however, its use on the nail unit is a very recent concept, yet to attain popularity. Chemical exfoliation and enhancement of penetration of topical antimycotics constitute the basic mechanism of nail peeling in ONM, whether one uses $50-70 \%$ GA or phenol peels. But only few peeling agents 
Fig. 1. The procedure of chemical peeling of nail. a The nail folds are occluded with vaseline before application of chemical peel. b Chemical peel is applied with the help of a paint brush followed by a second coat 20 min later.

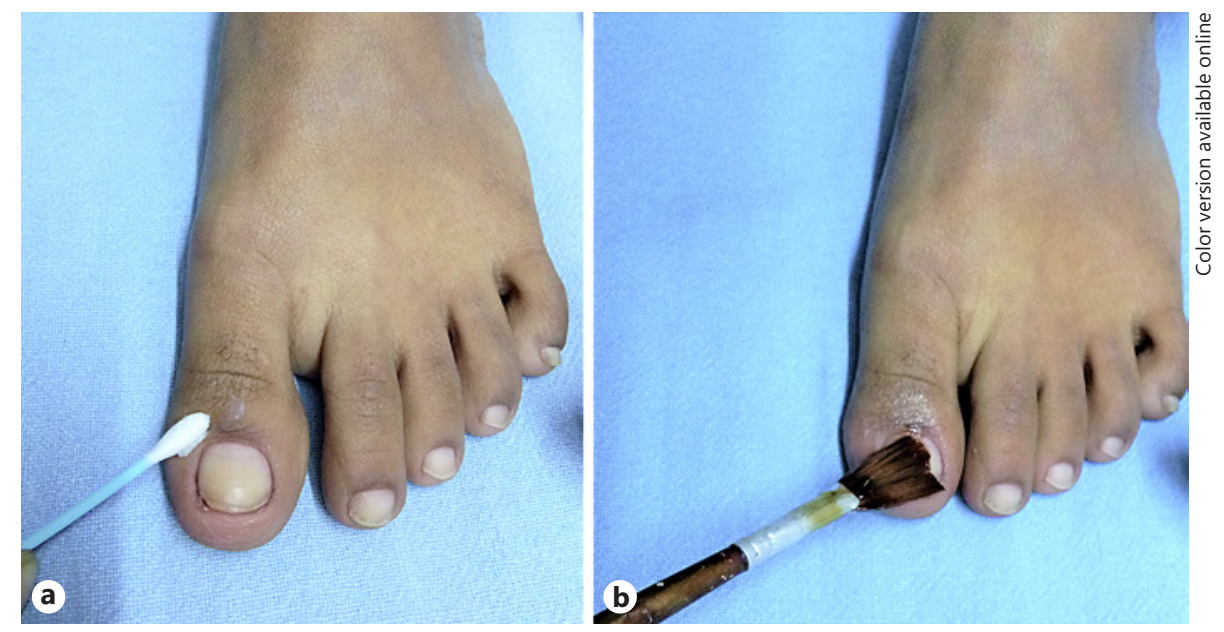

Patient 1

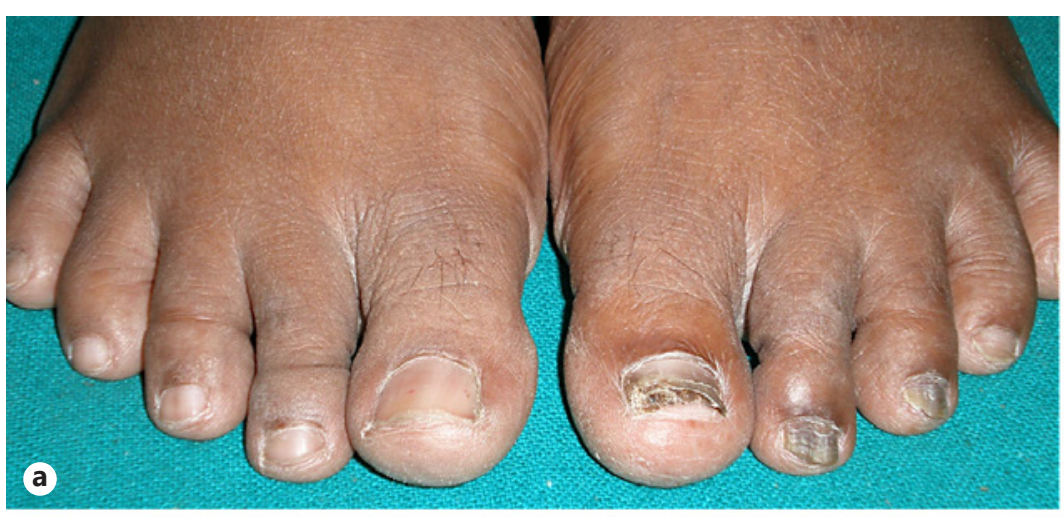

Patient 2
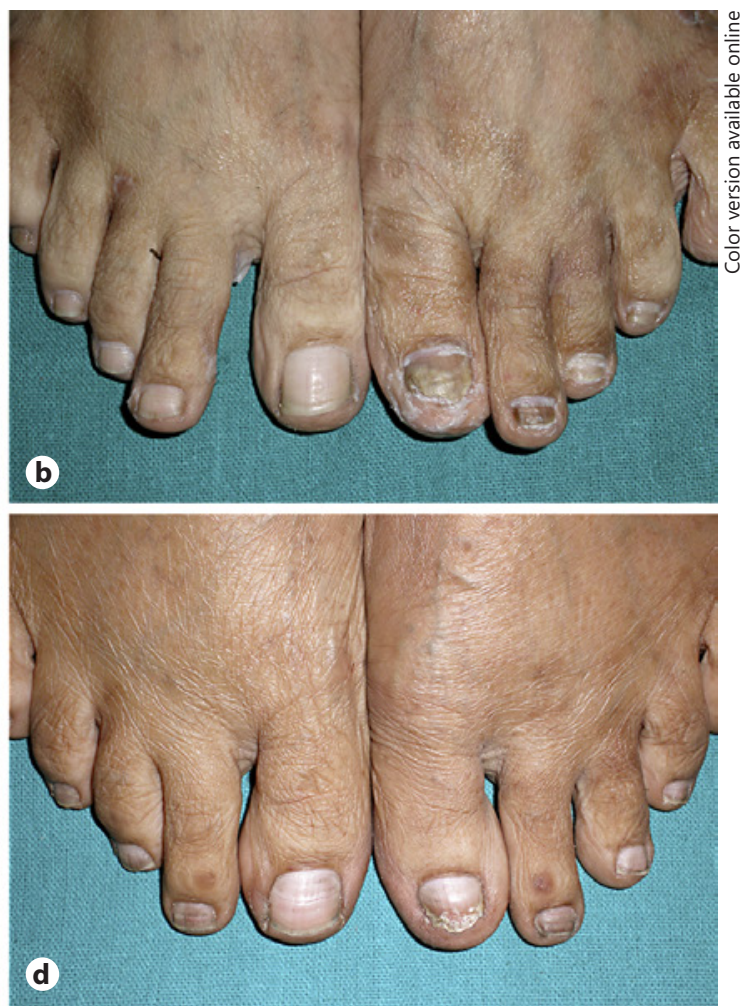

d

c

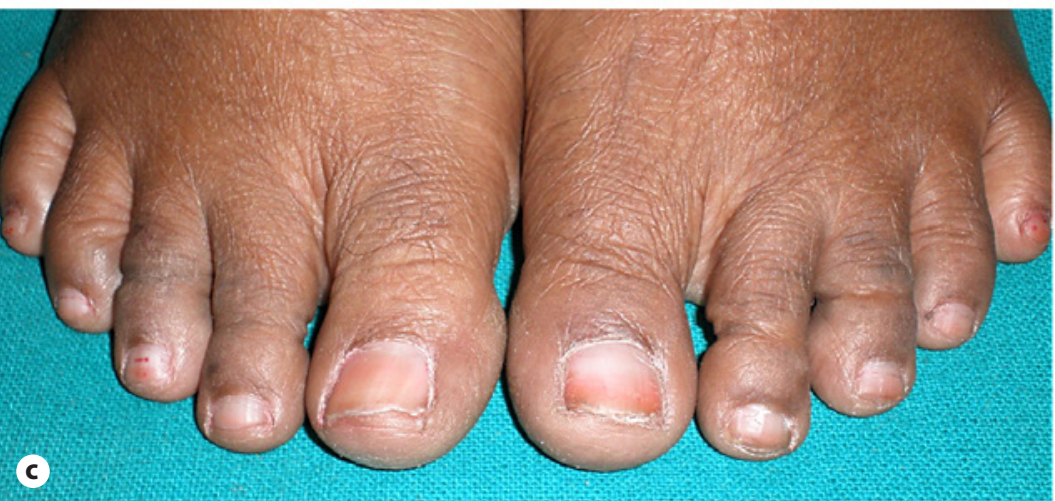

Fig. 2. Pre- (a, b) and post-peel results $(\mathbf{c}, \mathbf{d})$ showing marked improvement in onychomycotic nails.

(such as the ingredients of Black Peel) have additional specific antifungal and antibiofilm effects. Black peel is a popular cosmetic anti-acne peel (manufactured by THERADERM, South Korea and marketed in India by Aakaar Medical Technologies Pvt. Ltd.). BAA, derived from black rice has antimicrobial including antifungal activity and also breaks biofilm $[7,8]$. Salicylic acid enhances penetra-

Chemical Peeling for Onychomycosis tion of other peel agents and CPO [9]. In addition, SA also exerts direct antifungal and antibiofilm effects [10]. Jasmonic acid, a phytohormone, and its main derivative methyl-jasmonate (MeJa) are present in most of the plants as growth stimulators [11]. Although the antifungal activity of JA and MeJa against fungi pathogenic to plants such as Penicillium digitatum was discovered long back [12], its

Skin Appendage Disord 2019;5:181-185 DOI: $10.1159 / 000495152$ 
potent inhibitory activity against human pathogenic fungi including Candida spp. and Aspergillus niger has been reported relatively recently [13]. Purportedly, fatty acids of the jasmonate cascade formed by lipoxygenase-mediated peroxidation of linoleic acid participate in a lipid-based signalling system that induces the synthesis of specific proteinase inhibitors of fungi [11]. Sulphur has been a wellknown polyantimicrobial and antifungal agent, especially popular as a treatment for dermatophytosis in animals. Its antifungal properties and utility in human cutaneous mycosis have decent evidence [14]. The recent study on the beneficial impact of sulphur-rich thermal waters of Mamasani (South Iran) on many dermatoses including tinea versicolor and dermatophytic infections has reinforced its antifungal activity even in extreme dilutions [15]. The antibacterial and antifungal activities of sulphur are likely due to transformation of hydrogen sulfide (produced by sulphur-oxygen radicals interaction in deep epidermis) into pantothenic acid [15]. Topical potassium iodide exerts broad-spectrum antiseptic and disinfectant activity [16]. Although not directly toxic, KI probably exerts its cidal and antibiofilm effects after in-vivo conversion to iodine by neutrophils-generated myeloperoxidase $[17,18]$. It also potentiates the antibiofilm effect of PDT [19].

\section{Conclusion}

Thus, this pro-active repurposing of cosmetic ingredients like a specific chemical peel for a difficult-to-treat cutaneous/nail infection based on plausible and evidence- backed logic and facts has yielded an out-of-the box effective and safe treatment option for ONM in patients in whom treatment is essential, and oral antifungals have to be avoided. Combination with CPO undoubtedly contributes to the overall impact and provides early cure to the patients. The encouraging results of our two cases warrant exploration in a well-designed blinded randomized controlled trial.

\section{Statement of Ethics}

The authors have no ethical conflicts to disclose. Authors confirms that subjects have given their informed consent and that the study protocol has been approved by the institute's committee on human research.

\section{Disclosure Statement}

The authors have no conflicts of interest to declare.

\section{Author Contributions}

All authors have made substantial contributions to the conception or design of the work, or the acquisition, analysis, or interpretation of data for the work; and have been drafting the work or revising it critically for important intellectual content; and gave final approval of the version to be published; and agreed to be accountable for all aspects of the work in ensuring that questions related to the accuracy or integrity of any part of the work are appropriately investigated and resolved.

\section{References}

1 Gupta AK, Carviel J, Shear NH. Antibiofilm Treatment for Onychomycosis and Chronic Fungal Infections. Skin Appendage Disord. 2018 Aug;4(3):136-40.

2 Alberdi E, Gómez C. Efficiency of methylene blue-mediated photodynamic therapy vs intense pulsed light in the treatment of onychomycosis in the toenails. Photodermatol Photoimmunol Photomed. 2018 Aug; DOI: 10.1111/phpp.12420. Epub ahead of print.

3 Houang J, Perrone G, Mawad D, Boughton PC, Ruys AJ, Lauto A. Light treatments of nail fungal infections. J Biophotonics. 2018 Mar; 11(3):e201700350.

4 Zalacain A, Merlos A, Planell E, Cantadori EG, Vinuesa T, Viñas M. Clinical laser treatment of toenail onychomycoses. Lasers Med Sci. 2018 May;33(4):927-33.
5 Banga G, Patel K. Glycolic Acid peels for nail rejuvenation. J Cutan Aesthet Surg. 2014 OctDec;7(4):198-201.

6 Daulatabad D, Nanda S, Grover C. Intra-individual Right-left Comparative Study of Medium Depth Peels in Superficial Nail Abnormalities. J Cutan Aesthet Surg. 2017 Jan-Mar; 10(1):28-32.

7 de Castro RD, Mota AC, de Oliveira Lima E, Batista AU, de Araújo Oliveira J, Cavalcanti AL. Use of alcohol vinegar in the inhibition of Candida spp. and its effect on the physical properties of acrylic resins. BMC Oral Health. 2015 Apr;15(1):52.

8 Bjarnsholt T, Alhede M, Jensen PØ, Nielsen AK, Johansen HK, Homøe P, et al. Antibiofilm Properties of Acetic Acid. Adv Wound Care (New Rochelle). 2015 Jul;4(7):363-72.
9 Arif T. Salicylic acid as a peeling agent: a comprehensive review. Clin Cosmet Investig Dermatol. 2015 Aug;8:455-61.

10 Alem MA, Douglas LJ. Effects of aspirin and other nonsteroidal anti-inflammatory drugs on biofilms and planktonic cells of Candida albicans. Antimicrob Agents Chemother. 2004 Jan;48(1):41-7.

11 Martínez JA. Natural Fungicides Obtained from Plants, Fungicides for Plant and Animal Diseases. In: Dhanasekaran D, editor. Fungicides for Plant and Animal Diseases. 1st ed. Rijeka, Croatia: InTech; 2012. pp. 1-28. ISBN: 978-953-307-804-5, Available from http://www.intechopen.com/books/ fungicides-for-plant-and-animal-diseases / natural-fungicides-obtainedfromplants. 
12 Droby S, Porat RE, Weiss B, Shapiro B, Philosoph-Hadas S, Meir S. Suppressing green mold decay in grapefruit with postharvest jasmonate application. J Am Soc Hortic Sci. 1999;124(2):184-8.

13 Greene SL, Huang YE, Huang LD, Weart IF, Yang SA, Malik S, et al. (2011). Antimicrobial Compositions Comprising a Jasmonic Acid Compound as Active Agent. European Patent No. EP 1845964B1. Paris, France: European Patent Office.

14 Gupta AK, Nicol K. The use of sulfur in dermatology. J Drugs Dermatol. 2004 Jul-Aug; 3(4):427-31.
15 Hamidizadeh N, Simaeetabar S, Handjani F, Ranjbar S, Moghadam MG, Parvizi MM. Composition of minerals and trace elements at Mamasani thermal source: A possible preventive treatment for some skin diseases. J Educ Health Promot. 2017 Dec;6(1):110.

16 Costa RO, Macedo PM, Carvalhal A, Bernardes-Engemann AR. Use of potassium iodide in dermatology: updates on an old drug. An Bras Dermatol. 2013 May-Jun;88(3):396402.

17 Mahajan VK. Sporotrichosis: an overview and therapeutic options. Dermatol Res Pract. 2014;2014:272376
18 Masood R, Wrobel B, Dale H. A Comparison of the Effectiveness of Tincture of Iodine and potassium Iodide on Chronic Sinusitis $\mathrm{Pa}$ tients with Biofilm. J Neurol Neurosurg. 2017; 6(3):555690.

19 Wen X, Zhang X, Szewczyk G, El-Hussein A Huang YY, Sarna T, et al. Potassium Iodide Potentiates Antimicrobial Photodynamic Inactivation Mediated by Rose Bengal in In Vitro and In Vivo Studies. Antimicrob Agents Chemother. 2017 Jun;61(7):e00467-17. 